\title{
PPAR $\gamma$ and human metabolic disease
}

\author{
Robert K. Semple, ${ }^{1}$ V. Krishna K. Chatterjee, ${ }^{2}$ and Stephen O’Rahilly'
}

${ }^{1}$ Department of Clinical Biochemistry and 2Department of Medicine, University of Cambridge, Addenbrooke’s Hospital, Cambridge, United Kingdom.

\begin{abstract}
The nuclear receptor family of PPARs was named for the ability of the original member to induce hepatic peroxisome proliferation in mice in response to xenobiotic stimuli. However, studies on the action and structure of the 3 human PPAR isotypes (PPAR $\alpha$, PPAR $\delta$, and PPAR $\gamma$ ) suggest that these moieties are intimately involved in nutrient sensing and the regulation of carbohydrate and lipid metabolism. PPAR $\alpha$ and PPAR $\delta$ appear primarily to stimulate oxidative lipid metabolism, while PPAR $\gamma$ is principally involved in the cellular assimilation of lipids via anabolic pathways. Our understanding of the functions of PPAR $\gamma$ in humans has been increased by the clinical use of potent agonists and by the discovery of both rare and severely deleterious dominant-negative mutations leading to a stereotyped syndrome of partial lipodystrophy and severe insulin resistance, as well as more common sequence variants with a much smaller impact on receptor function. These may nevertheless have much greater significance for the public health burden of metabolic disease. This Review will focus on the role of PPAR $\gamma$ in human physiology, with specific reference to clinical pharmacological studies, and analysis of PPARG gene variants in the abnormal lipid and carbohydrate metabolism of the metabolic syndrome.
\end{abstract}

\section{Introduction}

PPAR $\alpha$ is the prototypic PPAR, mediating both peroxisome proliferation and tumorigenesis in the livers of mice exposed to xenobiotic substances (1). However, neither PPAR $\alpha$ nor its 2 closely related homologues PPAR $\gamma$ and PPAR $\delta$ are capable of supporting significant peroxisome proliferation in human liver, even in response to potent agonists. Instead, extensive experimental evidence now implicates these 3 nuclear hormone receptors in the regulation and coordination of lipid and carbohydrate metabolism (Table 1). Current models of PPAR $\alpha$ and PPAR $\delta$ function (and hence their potential relevance to human disease) rely heavily on data from cell culture and mouse studies. This Review summarizes the key roles of PPAR $y$ in the entraining of human intermediary metabolism to nutritional and other environmental cues.

PPAR $\gamma$ molecular structure and function. PPARs are members of the nuclear hormone receptor superfamily that bind to specific DNA response elements as heterodimers with the retinoid $\mathrm{X}$ receptor. Ligand binding leads to preferential recruitment of chromatin-decondensing coactivator complexes and favors dismissal of the corepressor complex. In addition, PPARs may influence gene expression indirectly, and usually negatively, through competition with other transcription factors. This appears to be particularly true for genes implicated in immunomodulatory effects of PPARs, including IFN- $\gamma$, repressed by PPAR $\gamma$ in T cells (2), and fibrinogen, repressed in hepatocytes by PPAR $\alpha$ via titration of the accessory molecule glucocorticoid receptor-interacting protein $1 /$ transcriptional intermediary factor 2 (GRIP1/TIF2) (3).

Insights from ligand studies. Growing evidence suggests that the identity of the bound ligand defines the repertoire of accessory molecules recruited by PPAR $\gamma$ and hence determines the transcriptional response. However, whether a physiologically relevant, high-affinity endogenous ligand for PPAR $\gamma$ exists is not clear. The most widely implicated candidate ligand is 15 -deoxy- $\Delta^{12,14}$-pros-

Nonstandard abbreviations used: FA, fatty acid; PLRS, PPAR $\gamma$ ligand resistance syndrome; TG, triglyceride; TZD, thiazolidinedione.

Conflict of interest: R.K. Semple and V.K.K. Chatterjee have no conflict of interest. S. O'Rahilly has received research grant support from GlaxoSmithKline, manufacturer of rosiglitazone.

Citation for this article: J. Clin. Invest. 116:581-589 (2006). doi:10.1172/JCI28003. taglandin J2. However, doubt about this ligand's role as the true endogenous ligand was raised when it was recently shown to be produced at extremely low levels during adipogenesis in vitro and when PPAR $\gamma$ activity is high in humans (4). Conversely, use of a PPAR $\gamma$ activity-sensing construct in 3T3-L1 preadipocytes has demonstrated the generation of an as-yet unidentified, highly active endogenous ligand during the early stages of adipogenesis (5). The ligand-binding pocket of PPAR $\gamma$ has a markedly open conformation, and various unsaturated fatty acids (FAs), oxidized lipid species, eicosanoids, and prostaglandins have been shown to activate the receptor with binding affinities in the micromolar range in vitro. This has led to the suggestion that PPAR $\gamma$ does not have a physiologically relevant specific ligand but rather acts as a generic "sensor" of the flux of FAs and related molecules, a property that would be in tune with a role for PPAR $\gamma$ in transducing nutritional signals into metabolic responses.

PPAR $\gamma$ tissue distribution and cellular role. The PPAR $\gamma$ gene produces 2 proteins, PPAR $\gamma 1$ and the nearly adipose-specific PPAR $\gamma 2$. PPAR $\gamma 2$ has 28 additional $N$-terminal amino acids that confer a 5 - to 6-fold increase in transcription-stimulating activity of the ligand-independent activation function-1 domain (6-8). Expression of PPAR $\gamma$ is highest in adipose tissue, where it is the key orchestrator of the transcriptional cascade underlying adipocyte differentiation, as established in vitro by gain-of-function (9-11) and loss-of-function experiments $(12,13)$, and by in vivo genetic manipulation $(14,15)$. PPAR $\gamma$ also plays a key role in the entraining of adipose tissue lipid metabolism to nutritional state. Its expression is highest postprandially (16), and its activation leads to upregulation of genes that mediate FA uptake and trapping (17-22) (Figure 1). PPAR $\gamma$ may also promote futile cycling in adipocytes between triglyceride (TG) esterification and de-esterification (23). However, the original observation of PPAR $\gamma$-mediated upregulation of glycerol kinase in human adipocytes was not replicated by a second study, which moreover failed to find any physiological evidence of such glycerol cycling in human adipose tissue in vivo (24).

Although the role of PPAR $\gamma$ in adipose tissue development and function is established, its low levels in tissues central to glucose homeostasis, including skeletal muscle, liver, and pancreatic $\beta$ cells, raise the question of its possible physiological and pharma- 


\section{Table 1}

Simplified overview of current understanding of the metabolic roles of the 3 PPAR isoforms

\begin{tabular}{|c|c|c|c|}
\hline & $\operatorname{PPAR} \alpha$ & $\operatorname{PPAR} \gamma$ & PPAR $\delta$ \\
\hline Sites of highest expression & Liver, kidney, heart & Adipose tissue, macrophages & $\begin{array}{l}\text { Adipose tissue, skin, brain, } \\
\text { but widespread }\end{array}$ \\
\hline Cellular processes activated & $\begin{array}{l}\text { Fatty acid } \beta \text {-oxidation, lipoprotein } \\
\text { synthesis, amino acid catabolism }\end{array}$ & $\begin{array}{l}\text { Adipocyte differentiation, } \\
\text { triglyceride synthesis }\end{array}$ & Fatty acid $\beta$-oxidation \\
\hline Physiological function & $\begin{array}{l}\text { Coordination of metabolic } \\
\text { response to fasting }\end{array}$ & $\begin{array}{l}\text { Differentiation of adipocytes, } \\
\text { FA trapping }\end{array}$ & Muscle fiber type determination? \\
\hline Examples of target genes & $\begin{array}{l}\text { Carnitine palmitoyl transferase I, } \\
\text { HMG CoA synthase 2, apoA-I }\end{array}$ & $\begin{array}{l}\text { Fatty acid-binding protein } 4, \\
\text { lipoprotein lipase, adiponectin }\end{array}$ & $\begin{array}{l}\text { Acyl-CoA oxidase, carnitine palmitoyl } \\
\text { transferase I }\end{array}$ \\
\hline $\begin{array}{l}\text { Metabolic phenotype } \\
\text { of knockout mice }\end{array}$ & $\begin{array}{l}\text { Fasting hypoglycemia, hypothermia, } \\
\text { hypoketonemia, and hepatic steatosis }\end{array}$ & $\begin{array}{l}\text {-/- Lethal, }-/+ \text { more insulin } \\
\text { sensitive at baseline }\end{array}$ & $\begin{array}{l}\text { Reduced base-line adiposity; increased } \\
\text { obesity on high-fat feeding }\end{array}$ \\
\hline
\end{tabular}

cological importance at those sites $(7,25,26)$. Furthermore, its high expression in macrophages, which are now known to infiltrate the dysfunctional adipose tissue of obese subjects $(27,28)$, may be pathologically important. However, the pathophysiological importance of PPAR $\gamma$ in these extra-adipose cells and tissues is not yet clear.

The determinants of the specific responses to PPAR $\gamma$ activation are also incompletely understood and cannot be completely accounted for by PPAR tissue distribution, intrinsic sequence specificity of PPAR response element-containing promoters, or expression of endogenous ligands. Tissue-specific or temporally regulated repertoires of accessory cofactor proteins may account for some variation in receptor activity, acting in combinatorial fashion to "soft-wire" different responses of nuclear receptors to the same ligand (29). Nevertheless, despite uncertainty about these basic aspects of PPAR $\gamma$ function, analysis of pharmacological and genetic gain and loss of function in human subjects provides insights into PPAR $\gamma$ roles in physiology.

\section{Clinical insights into PPAR $\gamma$ function: thiazolidinedione therapy}

A crucial early indication of the importance of PPAR $\gamma$ in human metabolism came in 1995, when it was identified as the cognate receptor for the thiazolidinedione (TZD) class of insulin-sensitizing drugs (30). TZDs subsequently proved beneficial as the first new class of insulin-sensitizing agents. Three potent and highly PPAR $\gamma$-selective TZDs have been used in large-scale clinical practice to date, although the prototype, troglitazone, was withdrawn due to idiosyncratic hepatotoxicity. Extensive clinical trial data have consistently shown that TZDs produce significant improvement in glycemic control. Hyperinsulinemic clamp studies confirm improved whole-body insulin sensitivity with all 3 agents (31-36), accounted for mostly by increased glucose disposal rates, although a minor suppression of hepatic glucose output has been reported $(37,38)$. However, clinical use of TZDs is significantly limited by the occurrence of fluid retention, hemodilution, and heart failure in up to $15 \%$ of patients (39). An interesting insight into the potential mechanisms of TZDinduced heart failure is provided by studies of genetically modified mice in which PPAR $\gamma$ was deleted specifically in the renal tubule. These mice do not show the sodium retention seen in wild-type mice given TZDs, which implies that renal PPAR $\gamma$ has a direct role in renal tubular sodium handling $(40,41)$.

\section{Sites of action of TZDs in humans}

$T Z D$ action and PPARY in adipose tissue. In view of its well established role in adipogenesis, insulin sensitization by PPAR $\gamma$ activation could be interpreted as a direct result of the ability of PPAR $\gamma$ to expand adipose tissue. However, counterintuitively, TZDs are predominantly prescribed to enhance insulin sensitivity in patients with type 2 diabetes mellitus, which is often precipitated by the development of excess adipose tissue. This paradox may be partly explained by the depot-selective responses of adipose tissue to PPAR $\gamma$ activation. Indeed, in humans, treatment with TZDs leads to selective accumulation of subcutaneous adipose tissue (reviewed in ref. 42), with concomitant lack of change or reduction in the adiposity of visceral depots. Furthermore, some but not all ex vivo studies of preadipocyte differentiation have shown that abdominal subcutaneous preadipocytes differentiate in response to TZDs more readily than cells from visceral depots of the same subjects (43-45). It is not yet known whether TZD treatment increases subcutaneous fat mass in all body regions equally, a question that is of interest in light of the burgeoning evidence of important functional metabolic differences between upper-body (abdominal) and lower-body (including femoro-gluteal) subcutaneous fat $(46,47)$.

One hypothesis to explain the insulin-sensitizing effects of TZDs holds that this adipose remodeling enhances insulin sensitivity by favoring lipid accretion in depots that are less hormonally sensitive and are without direct access to the portal circulation and hence the liver. However, in addition to changing the gross topography of adipose tissue, PPAR $\gamma$ activation also alters adipocyte function in murine and cellular studies (Figure 1). If the effects of TZDs on genes related to FA trapping are also seen in humans, then TZDs are likely to act on adipose tissue to enhance its capacity to act as a sump for dietary FAs, safely sequestering them in adipocytes and partitioning them away from other insulin-sensitive tissues such as skeletal muscle. This has become known as the "lipid steal" hypothesis and has recently been supported by direct observation in rodents (48). Consistent with this model, TZDs have almost universally lowered fasting FFA levels in clinical trials $(32,34,35,49)$.

Another mechanism whereby TZD action on adipose tissue may influence insulin sensitivity in distant insulin-sensitive organs is by modifying the profile of hormones secreted from adipose tissue. Only recently has the capacity of adipose tissue to produce a variety of small molecules with autocrine, paracrine, or endocrine activity been appreciated. Of these human "adipocytokines" described to date, adiponectin appears to be the best-characterized 


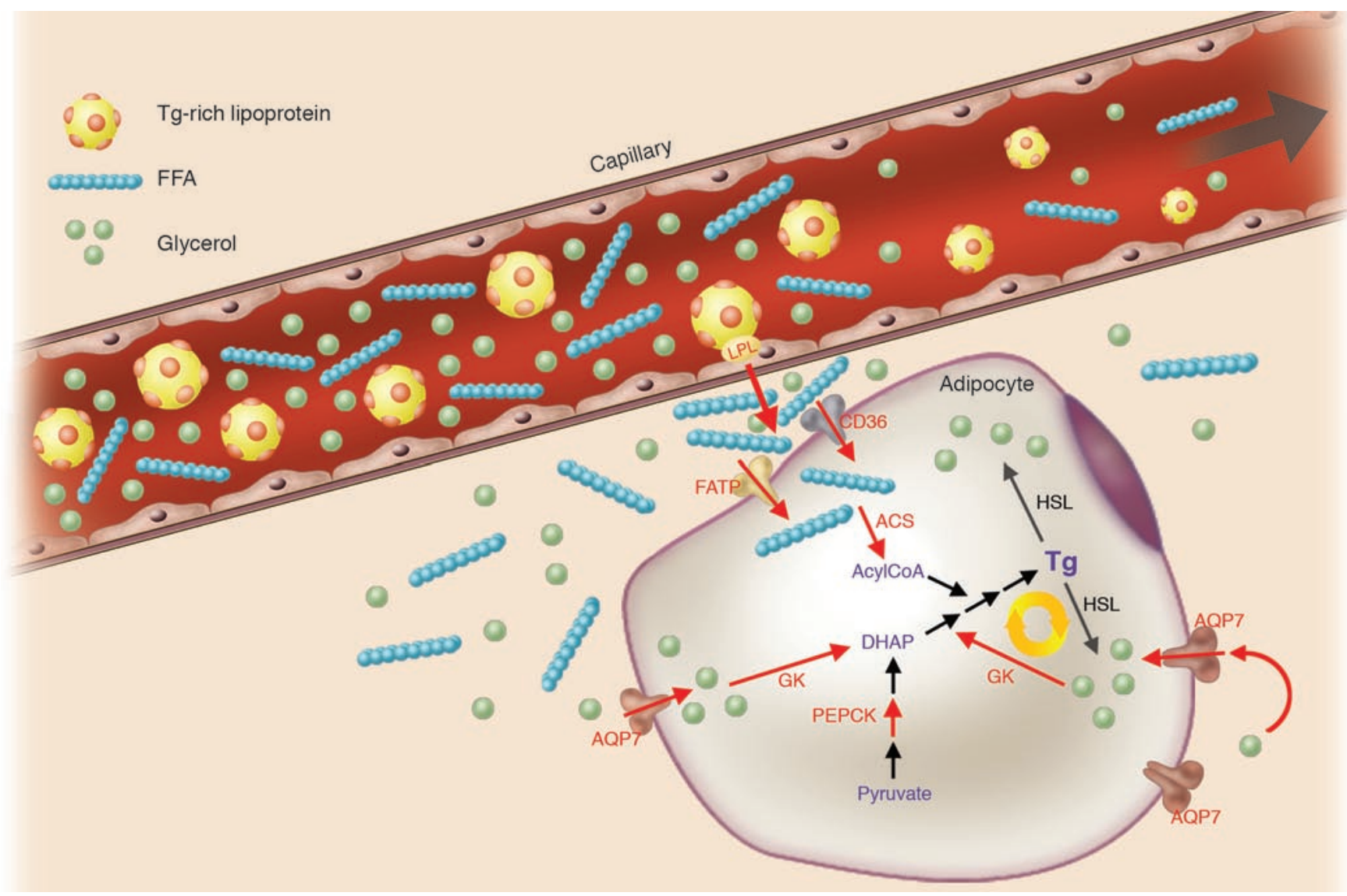

Figure 1

Actions of PPAR $\gamma$ in adipocyte FA trapping. PPAR $\gamma$ upregulates the transcription of genes involved in most stages of FA trapping, some of which are shown here in red. PPAR y enhances lipoprotein triglyceride hydrolysis by endothelial lipoprotein lipase (LPL) and uptake of free fatty acids (FFA) into adipocytes through fatty acid transport protein (FATP) and CD36. PPAR $\gamma$ also modifies triglyceride synthesis, starting with FFA esterification by Acyl CoA synthase. The futile glycerol cycle, recently proposed to be stimulated by PPAR , is illustrated by the yellow circular arrows, and depends upon glycerol kinase (GK), with aquaporin 7 (AQP7) as the adipocyte glycerol channel. The collective PPAR $\gamma$ actions reduce FFA levels in the venous effluent from adipose tissue, thereby limiting the lipotoxicity of other insulin-sensitive tissues that could result from FFA exposure. PEPCK, phosphoenolpyruvate carboxykinase; HSL, hormone-sensitive lipase; DHAP, dihydroxyacetone phosphate.

candidate to mediate the insulin-sensitizing effects of TZDs. Adiponectin is a multimeric complement-like protein that is among the most abundant plasma proteins (reviewed in ref. 50). In fact, adiponectin plasma levels correlate directly with insulin sensitivity (51-53) and inversely with adipose tissue mass $(54,55)$. Furthermore, TZDs increase adiponectin gene expression and plasma protein levels $(56,57)$, and expression of a hyperactive mutant adiponectin in mice results in adipose tissue redistribution and insulin sensitization that are akin to those seen with TZD treatment (58). However, despite this correlation between TZD-mediated insulin sensitization and the elevation of plasma adiponectin, direct evidence for insulin sensitization by adiponectin itself is lacking to date in humans. A further adipocytokine recently studied as a possible mediator of TZD action is retinol-binding protein 4 (RBP4) (59). In rodents, this inducer of insulin resistance is downregulated in adipose tissue on treatment with TZDs (59), but formal study of the effect of TZDs on RBP4 in humans is awaited.

TZD action and PPARY in skeletal muscle. Although the level of PPAR $\gamma$ protein is relatively low in skeletal muscle (25), the total mass of muscle and its function as the site of $70 \%$ of insulin-medi- ated glucose disposal in humans suggest that physiologically important effects of PPAR $\gamma$ are plausible. However, although TZD treatment has consistently been found to enhance insulin-stimulated glucose uptake, muscle-autonomous roles of PPAR $\gamma$ are difficult to dissect out in vivo because of the complex and continuous metabolic cross-talk between different insulin-sensitive organs. In vitro, TZDs have been reported to enhance insulin-stimulated glucose uptake into cultured human skeletal muscle cells, mediated by enhancement of insulin-stimulated PI3Kactivity and translocation of GLUT4 (60-64), but the physiological importance of PPAR $\gamma$ in muscle remains unclear. Two independent reports of mice with muscle-specific PPAR $\gamma$ deletion produced discordant results. The first study reported modest whole-body insulin resistance and normal glucose disposal into muscle (65), while the second study reported progressive and severe insulin resistance due to markedly impaired muscle glucose uptake in response to insulin (66). These inconsistencies, while most likely attributable to methodological differences, cannot be accounted for at present.

TZD action and PPAR in the liver. TZD administration has been found to reduce hepatic glucose output in some $(37,38)$, but not 
A

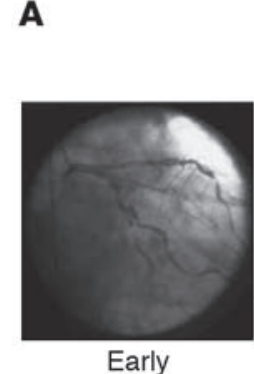
atherosclerosis

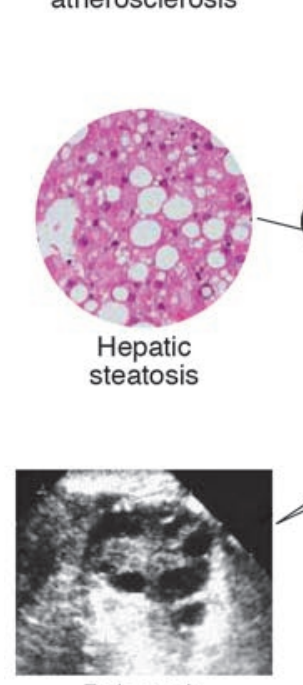

Polycystic ovaries
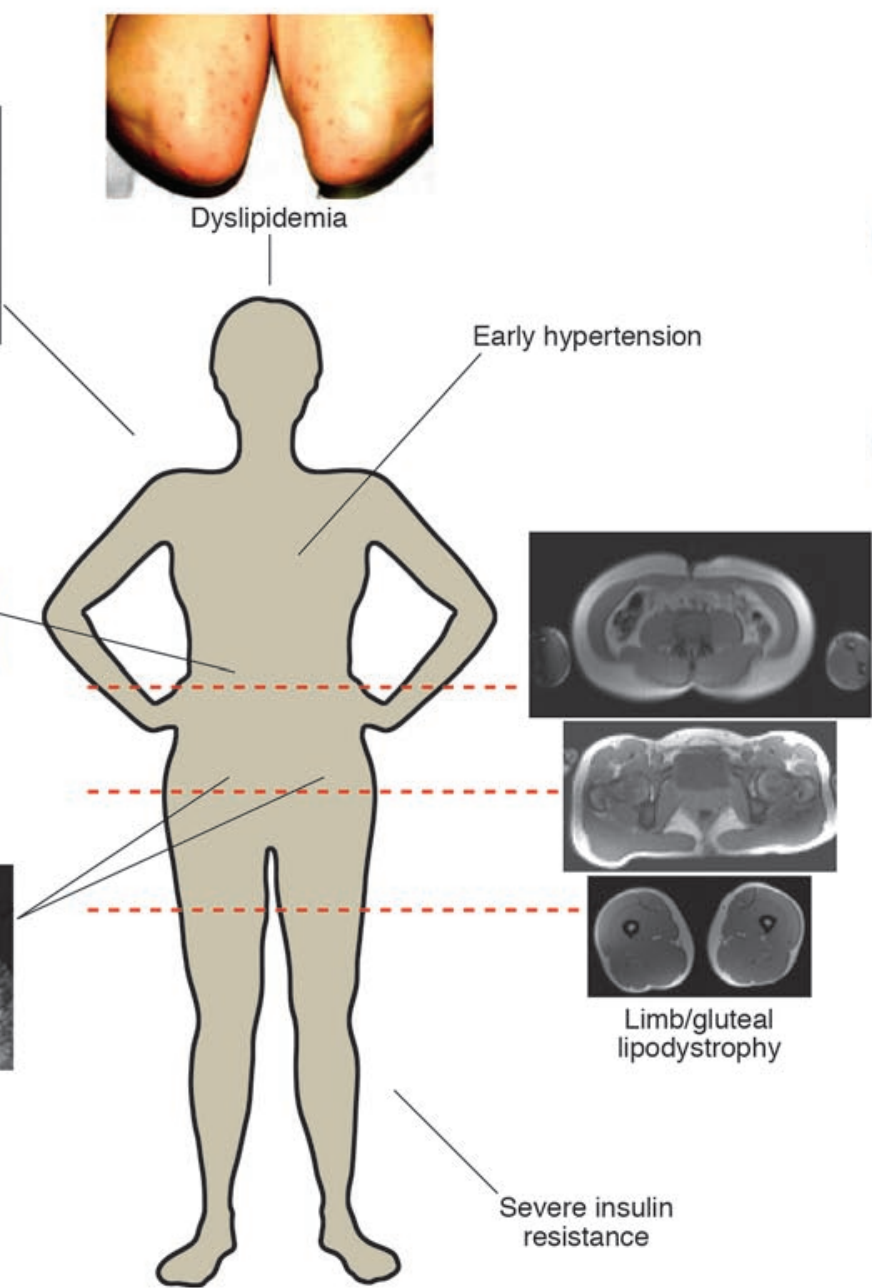

B

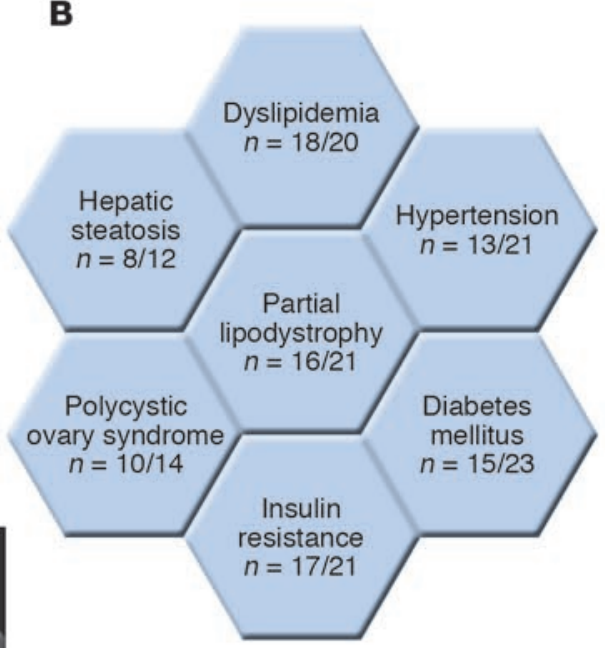

Figure 2

PPAR $\gamma$ ligand resistance syndrome (PLRS). (A) Central clinical features. A 41-year-old woman with a heterozygous dominant-negative C131Y PPAR $y$ mutation is pictured. Presentation was with severe hypertension and recurrent hypoglycemia at 20 years old. Representative MRI sections are shown illustrating femoro-gluteal lipodystrophy. (B) Overview of phenotypic characteristics of PLRS subjects. The denominator of $n$ refers to the number of subjects for whom information is available. all (31), clinical studies to date, and only low levels (10-30\% of adipose tissue) of PPAR $\gamma$ mRNA expression in human liver have been reported $(7,26)$. However, in many rodent models of diabetes and insulin resistance with hepatic steatosis, encompassing both lipoatrophy and hyperphagic obesity, hepatic expression of PPAR $\gamma$ is markedly elevated $(16,67-71)$. Moreover, adenovirally mediated hepatic overexpression of PPAR $\gamma$ in mice (72) leads to hepatic steatosis with upregulation of a wide range of PPAR $\gamma$-responsive genes generally considered to be adipocyte specific, such as adipsin, adiponectin, and fatty acid-binding protein 4 . Nonetheless, there are no data demonstrating increased PPAR $\gamma$ expression in human hepatic steatosis. Indeed, accumulating evidence suggests that TZD treatment reduces nonalcoholic hepatic steatosis in humans (73-75), suggesting that, even if PPAR $\gamma$ is a primary mediator of hepatic lipid accumulation, this propensity to assimilate lipid is quantitatively less important than the beneficial effects of TZDs on FA trapping in adipose tissue.

\section{PPAR $\gamma$ ligand resistance syndrome}

In the absence of potent pharmacological antagonists of PPAR $\gamma$ for use in humans, study of the effect of PPAR $\gamma$ loss of function relies instead on the natural occurrence of mutant PPAR $\gamma$ alleles that impair native PPAR $\gamma$ function. To date, 3 groups have independently reported loss-of-function mutations in the ligand- binding domain of human PPAR $\gamma$ (76-78). Some of these mutant receptors exert a dominant-negative effect, due to aberrantly high affinity for corepressor molecules and attenuated recruitment of coactivators (76-79). Together these reports describe 8 adult subjects from 4 pedigrees, and through continued screening of the PPARG gene in subjects with severe insulin resistance, we have identified a further 15 subjects from 8 kindreds who harbor dominant-negative mutant PPAR $\gamma$ alleles that impair PPAR $\gamma$ function by greater than $50 \%$ in vitro and ex vivo (V.K.K. Chatterjee and S. O'Rahilly, unpublished observations).

An overview of the phenotype of these subjects is presented in Figure 2A. A stereotyped form of partial lipodystrophy is seen in a majority of subjects (Figure 2B), with loss of subcutaneous fat from the limbs and gluteal region, and relative preservation of both subcutaneous and visceral abdominal depots (Figure 2A). This contrasts with the pattern of adipose tissue loss seen in the most common inherited partial lipodystrophy, due to mutations in the gene encoding lamin $\mathrm{A} / \mathrm{C}$, which includes abdominal subcutaneous and mammary depots, with compensatory adiposity in the supraclavicular and neck region. The PPAR $\gamma$ ligand resistance syndrome (PLRS) subjects without apparent lipodystrophy are either prepubertal or physically fit with low total-body adiposity, both situations in which relative diminution of different depots may be difficult to discern. Furthermore, the lipodystrophy is subtle in other 
subjects, and total-body adiposity may be substantial. The depotselective loss of adipose tissue is concordant with studies of TZD action $(35,80,81)$ and is broadly in keeping with the role of PPAR $\gamma$ as the prime regulator of adipogenesis. However, it also speaks to substantial heterogeneity even among subcutaneous preadipocytes from different parts of the body. There is insufficient information available about differences in subcutaneous adipose tissue from different anatomical regions (for example, abdominal versus femoro-gluteal) to explain the selective loss of femoro-gluteal fat in terms of differences of gene expression or functional adipocyte biology at present. Nevertheless, the importance of femoro-gluteal adipose depots in normal metabolism is further supported by the severe insulin resistance seen in other genetic and acquired forms of lipodystrophy that affect these depots, as opposed to the fairly mild metabolic derangement seen in acquired partial lipodystrophy, which generally spares regions below the umbilicus (82).

Like those with other types of total or caudal lipodystrophy, the majority of the PLRS subjects also have profound metabolic derangements in the form of severe insulin resistance and earlyonset diabetes mellitus (mean age of diagnosis 31 years, range 8-53 years), with polycystic ovary syndrome seen in female subjects as a direct consequence of insulin resistance (Figure 2B). Most subjects have marked, sometimes extreme, dyslipidemia characterized by high TG and low HDL cholesterol levels (V.K.K. Chatterjee and S. O'Rahilly, unpublished observations; and refs. 77, 78, 83) (Figure $2 \mathrm{~B})$. The prevailing model to explain insulin resistance in human syndromes of lipodystrophy invokes an insufficient adipose capacity to buffer dietary FAs, with consequent lipotoxicity due to deposition of TG and acyl-CoA in insulin-sensitive tissues. This lipotoxicity is exacerbated in those with total, and in a proportion of those with partial, lipodystrophy by leptin deficiency that leads to hyperphagia and increased caloric intake, and possibly loss of direct peripheral actions of leptin.

Given the fairly modest degree of lipodystrophy exhibited by some PLRS subjects, the question of whether there is also dysfunction of the residual adipose tissue arises. Several lines of evidence suggest that this is indeed the case. First, PLRS subjects often exhibit extreme hypertriglyceridemia that is out of keeping with only mild, if any, diminution of adipose tissue, as evidenced by direct determination or the surrogate marker of plasma leptin level. For example, in one subject a single high-fat meal was sufficient to result in severe fasting hypertriglyceridemia and acute diabetes despite a total body fat content of $23 \%$, within the normal adult female range (84). Second, direct measurement of adipose tissue TG trapping in a single subject with the PPAR $\gamma$ P467L mutation revealed it to be grossly reduced (83). This early evidence suggests that PLRS includes adipose tissue dysfunction, which may be pathogenically more significant than the overall degree of lipodystrophy observed. Further evidence of this may come from comparison of TG clearance per unit mass of adipose tissue in healthy subjects, in those with PLRS, and in those with lamin A/C mutations, in whom lipodystrophy is believed to have a primarily structural basis, with no reason to suppose a functional deficit in the residual adipocytes.

Some limited conclusions about the role of PPAR $\gamma$ in other insulin-sensitive tissues can also be drawn on the basis of these subjects. First, the common and significant hepatic steatosis (progressing to cirrhosis in 1 subject) suggests that, even if hepatic PPAR $\gamma$ expression is upregulated in the context of steatosis, wildtype receptor activity is not required for TG accumulation. Thus any loss of PPAR $\gamma$ function in the liver is offset by the greater func- tional receptor deficit in white adipose tissue, in line with the beneficial effects of TZDs on nonalcoholic steatohepatitis (73-75).

Inferences about the importance of PPAR $\gamma$ in muscle are more difficult to make based on the available data. However, intramyocellular TG levels in 2 severely insulin-resistant subjects with dominant-negative loss-of-function mutations surprisingly showed no elevation compared with those in healthy controls, despite significant muscle insulin resistance as established by hyperinsulinemic euglycemic clamp studies (83). This observation, if it holds true on further study, implies that simple accumulation of intramyocellular lipid due to impaired adipose tissue FA trapping cannot explain the severe muscle insulin resistance, and it suggests that a direct effect of PPAR $\gamma$ on muscle insulin sensitivity, or an effect mediated by altered levels of circulating adipocytokines, may need to be invoked.

Early-onset hypertension has also persisted as a cardinal feature of PLRS with the definition of more cases (V.K.K. Chatterjee and S. O'Rahilly, unpublished observations) (Figure 2B) and sets it apart from other types of inherited and acquired lipodystrophy, although the mechanism remains uncertain. In view of the propensity of PPAR $\gamma$ activation by TZDs to increase blood volume, it may be that hypertension in PLRS is driven primarily by increased vascular tone. Consistent with this hypothesis, PPAR $\gamma$ is expressed in both vascular smooth muscle and endothelial cells and may stimulate muscle relaxation through inhibition of vascular calcium channels (85), and through reduction of levels of vasoactive peptides such as endothelin-1 (86). Interestingly, transgenic mice expressing dominant-negative P465L PPAR $\gamma$, although they showed no insulin resistance, were found to be hypertensive (87). This not only provides supportive evidence for a pathogenic role for dominant-negative PPAR $\gamma$ mutations in hypertension but also suggests that the mechanisms involved are distinct from those leading to insulin resistance. The severe form of the metabolic syndrome represented by PLRS would be expected to accelerate atherogenesis. Consistent with this hypothesis, early-onset coronary artery disease is evident in some young affected subjects in this cohort. This emerging vascular phenotype suggests that, in addition to cardiovascular risk factors, macrophage PPAR $\gamma$ dysfunction might contribute to accelerated atherogenesis.

The phenotypic variation within affected PLRS kindreds may be striking, with male subjects and individuals with high levels of aerobic exercise demonstrating mitigated deleterious metabolic consequences of the PPAR $\gamma$ mutations. This evidence of important environmental and possibly background genetic modifiers of the PLRS phenotype is in accord with the studies of the PPAR $\gamma$ Pro12Ala polymorphism (discussed below) and perhaps explains the metabolic phenotype of transgenic mice harboring dominant-negative PPAR $\gamma$ alleles $(87,88)$, which is much milder than that of PLRS subjects.

Deductions about the physiological function of PPAR $\gamma$ derived from phenotypes of subjects with dominant-negative mutations are based on the assumption that these mutations cause pure PPAR $\gamma$ loss of function. In fact, 2 naturally occurring mutations have been shown in vitro also to inhibit wild-type PPAR $\alpha$ and PPAR $\delta$ function (89), and although this has yet to be formally tested in vivo, this observation introduces a caveat to such extrapolation. A much cleaner example of PPAR $\gamma$ loss of function is afforded by the discovery of a frameshift mutation (null allele) in a single kindred with severe insulin resistance (90). The metabolic phenotype did not cosegregate with the PPAR $\gamma$ mutation in this pedigree, but a premature stop (nonsense mutation) in the muscle-specific glycogen-targeting subunit of protein phosphatase 1 (PPP1R3A) was later found in 
the same group of subjects. Although this too failed to cosegregate completely with the abnormal phenotype, double heterozygosity for the 2 gene defects occurred only in subjects with extreme insulin resistance. This kindred provides the first example of digenic insulin resistance and diabetes and may allow us to define a paradigm for the molecular pathogenesis of common type 2 diabetes mellitus, in which mild oligo- or polygenic defects in remote insulin-sensitive tissues interact to produce the systemic metabolic defect. Interestingly, the 3 subjects with heterozygosity for the PPAR $\gamma$ frameshift mutation alone had fasting insulin levels that were toward the bottom of the normal range, suggesting that haploinsufficiency for PPAR $\gamma$ alone does not lead to severe insulin resistance in the absence of a "second hit" on glucose homeostasis, consistent with findings in heterozygous PPAR $\gamma$-null mice $(91,92)$.

\section{Common variants in PPAR $\gamma$ and human metabolic disease}

The receptor mutations described hitherto are rare, and although they have profound phenotypic effects in affected individuals, they make a negligible contribution to the risk of insulin resistance or type 2 diabetes in the general population. In contrast, by far the most prevalent human PPAR $\gamma$ genetic variant is a polymorphism replacing alanine with proline at codon 12 (Pro12Ala) in the unique PPAR $\gamma 2$ amino-terminal domain, with an allelic frequency ranging between $2 \%$ and $23 \%$ in different ethnic groups $(93,94)$. In vitro, the Pro12Ala variant exhibits reduced binding to DNA and modest impairment of transcriptional activation $(95,96)$.

The methodology of genetic epidemiology has been extensively used to seek association between metabolic disease and Pro12Ala. Although early studies of type 2 diabetes were inconsistent, a metaanalysis in 2000 confirmed a modest (1.25-fold) but significant $(P=0.002)$ increase in diabetes risk with the Pro allele (93). Thus, if an entire population carried the Ala allele, the global prevalence of type 2 diabetes mellitus would be reduced by $25 \%$, making PPAR $\gamma$ potentially the most important common "diabetogene" thus far discovered. Subsequent reports in different ethnic groups have supported this finding (97-100). The failure of several reports to corroborate the association may be due to insufficient sample sizes and the influence of some of the modifying factors discussed below (101-104).

In the index study, carriers of the Ala polymorphism had a significantly lower BMI, and after correction for this, there was no difference in insulin sensitivity between genotypes (95). This observation, in conjunction with the lower transcriptional activity of the Ala variant in vitro, led to the hypothesis that improved insulin sensitivity might be accounted for entirely by changes in adiposity. However, subsequent studies have failed to yield consistent findings, with some demonstrating a modestly greater BMI in carriers of the Ala allele (105-107). A recent meta-analysis has further suggested a significant correlation of the Pro12Ala allele with higher $\mathrm{BMI}$ in those with a BMI above $27 \mathrm{~kg} / \mathrm{m}^{2}$. Thus, the influence of the Pro12Ala allele on total adiposity remains uncertain, and more subtle effects on adipose tissue topography have yet to be addressed.

Discrepancies in epidemiological studies may arise from the presence of linked sequence variants of functional significance in the PPAR $\gamma$ gene, some of which may oppose the effect of Pro12Ala (108). However, only a limited number of studies have examined haplotype associations with metabolic parameters $(109,110)$. Also likely to be of crucial importance are environmental modifiers of the effects of genetic variation in PPAR $\gamma$. The most frequently tested modifiers have been diet and aerobic exercise. However, although only Pro/Pro homozygotes were found in 2 studies to show a relationship between fat intake and $\mathrm{BMI}(111,112)$, other studies have shown greater sensitivity of Ala carriers to dietary factors. This has been evidenced by a stronger relationship between BMI and the ratio of dietary polyunsaturated fat to saturated fat intake (113), as well as by faster weight regain after a hypocaloric diet (114), and by a greater plasma TG response to $\omega 3$ FAs in Ala carriers compared with Pro homozygotes (115). Studies of differential responses to exercise have been more consistent, with Ala carriers proving more sensitive to aerobic exercise in terms of a range of different metabolic parameters or body weight (116-119), while one study found a greater interaction between dietary FA profile, exercise, and fasting insulin in Ala carriers than in Pro homozygotes (122).

The mechanism whereby this polymorphism affects glucose and lipid homeostasis remains to be fully determined. While some studies report enhanced suppression of lipolysis and hence adipose lipid trapping in Ala carriers $(121,122)$, others have detected no difference in fasting FFAs (122) or response to oral lipid (103), while another actually found lower post-heparin plasma lipoprotein lipase activity in Ala carriers (123). Although differences in adipocytokine levels are an attractive explanation for genotypespecific metabolic responses, the reported relationship of Pro12Ala genotype to plasma adiponectin has not been consistent (124, 125). In summary, common sequence variation in the PPAR $\gamma$ gene may account for a significant part of the population attributable risk for type 2 diabetes mellitus. Findings to date are supportive of a role for PPAR $\gamma$ at the interface of environment and the control of metabolism. However, further studies incorporating rigorous controls for genetic and environmental confounders are required to identify the molecular mechanisms involved.

\section{Conclusions}

PPAR $\gamma$ was first identified as a member of the nuclear receptor family just over a decade ago. The tremendous strides that have been made in understanding and manipulating its biology are a testament to the power of modern molecular biology and clinical medicine. However, we still have much to learn about the role of this molecule in human metabolic disease and its utility as therapeutic target. What is the "real" ligand, if such an entity exists? Would selective modulators of PPAR $\gamma$ interaction with particular coactivators or corepressors have therapeutic advantages? What are the long-term consequences of pharmacological PPAR activation for the progress of atherosclerosis? Can we exploit knowledge of dietary constituents that modulate PPAR $\gamma$ function to enhance health through a "nutrigenomic" approach? We anticipate considerable progress in addressing these questions but are sure that the next decade of research will hold as many surprises as the last.

\section{Acknowledgments}

V.K.K. Chatterjee and S. O'Rahilly are supported by the Wellcome Trust.

Address correspondence to: S. O'Rahilly, Department of Clinical Biochemistry, University of Cambridge, Addenbrooke's Hospital, Hills Road, Cambridge CB2 2QQ, United Kingdom. Phone: 44122-333-6855; Fax: 44-122-333-0598; E-mail: so104@medschl. cam.ac.uk. Or to: V.K.K. Chatterjee, Department of Medicine, University of Cambridge, Addenbrooke's Hospital, Hills Road, Cambridge CB2 2QQ, United Kingdom. Phone: 44-1223-336-842; Fax: 44-1223-336-846; E-mail: kkc1@mole.bio.cam.ac.uk. 
1. Issemann, I., and Green, S. 1990. Activation of a member of the steroid hormone receptor superfamily by peroxisome proliferators. Proc. Natl. Acad. Sci.U.S. A. 47:645-650.

2. Cunard, R., et al. 2004. Repression of IFN-gamma expression by peroxisome proliferator-activated receptor gamma. J. Immunol. 172:7530-7536.

3. Gervois, P., et al. 2001. Negative regulation of human fibrinogen gene expression by peroxisome proliferator-activated receptor alpha agonists via inhibition of CCAAT box/enhancer-binding protein beta. J. Biol. Chem. 276:33471-33477.

4. Bell-Parikh, L.C., et al. 2003. Biosynthesis of 15-deoxy-812,14-PGJ2 and the ligation of PPAR $\gamma$. J. Clin. Invest. 112:945-955. doi:10.1172/ JCI200318012.

5. Tzameli, I., et al. 2004. Regulated production of a peroxisome proliferator-activated receptorgamma ligand during an early phase of adipocyte differentiation in 3T3-L1 adipocytes. J. Biol. Chem. 279:36093-36102.

6. Tontonoz, P., Hu, E., Graves, R.A., Budavari, A.I., and Spiegelman, B.M. 1994. mPPAR gamma 2: tissue-specific regulator of an adipocyte enhancer. Genes Dev. 8:1224-1234.

7. Vidal-Puig, A.J., et al. 1997. Peroxisome proliferator-activated receptor gene expression in human tissues. Effects of obesity, weight loss, and regulation by insulin and glucocorticoids. J. Clin. Invest. 99:2416-2422.

8. Fajas, L., et al. 1997. The organization, promoter analysis, and expression of the human PPARgamma gene. J. Biol. Chem. 272:18779-18789.

9. Tontonoz, P., Hu, E., and Spiegelman, B.M. 1994 Stimulation of adipogenesis in fibroblasts by PPAR gamma 2, a lipid-activated transcription factor. Cell. 79:1147-1156.

10. Sandouk, T., Reda, D., and Hofmann, C. 1993. Antidiabetic agent pioglitazone enhances adipocyte differentiation of 3T3-F442A cells. Am. J. Physiol. 264:C1600-C1608.

11. Kletzien, R.F., Clarke, S.D., and Ulrich, R.G. 1992. Enhancement of adipocyte differentiation by an insulin-sensitizing agent. Mol. Pharmacol. 41:393-398.

12. Ren, D., Collingwood, T.N., Rebar, E.J., Wolffe, A.P., and Camp, H.S. 2002. PPARgamma knockdown by engineered transcription factors: exogenous PPARgamma2 but not PPARgamma1 reactivates adipogenesis. Genes Dev. 16:27-32.

13. Mueller, E., et al. 2002. Genetic analysis of adipogenesis through peroxisome proliferator-activated receptor gamma isoforms. J. Biol. Chem. 277:41925-41930.

14. Barak, Y., et al. 1999. PPAR gamma is required for placental, cardiac, and adipose tissue development. Mol. Cell. 4:585-595.

15. Rosen, E.D., et al. 1999. PPAR gamma is required for the differentiation of adipose tissue in vivo and in vitro. Mol. Cell. 4:611-617.

16. Vidal-Puig, A., et al. 1996. Regulation of PPAR $\gamma$ gene expression by nutrition and obesity in rodents. J. Clin. Invest. 97:2553-2561.

17. Schoonjans, K., et al. 1996. PPARalpha and PPARgamma activators direct a distinct tissue-specific transcriptional response via a PPRE in the lipoprotein lipase gene. EMBOJ. 15:5336-5348.

18. Motojima, K., Passilly, P., Peters, J.M., Gonzalez, F.J., and Latruffe, N. 1998. Expression of putative FA transporter genes are regulated by peroxisome proliferator-activated receptor alpha and gamma activators in a tissue- and inducer-specific manner. J. Biol. Chem. 273:16710-16714.

19. Tordjman, J., et al. 2003. Thiazolidinediones block FA release by inducing glyceroneogenesis in fat cells. J. Biol. Chem. 278:18785-18790.

20. Tontonoz, P., Hu, E., Devine, J., Beale, E.G., and Spiegelman, B.M. 1995. PPAR gamma 2 regulates adipose expression of the phosphoenolpyruvate carboxykinase gene. Mol. Cell. Biol. 15:351-357.

21. Olswang, Y., et al. 2002. A mutation in the peroxisome proliferator-activated receptor gamma-binding site in the gene for the cytosolic form of phosphoenolpyruvate carboxykinase reduces adipose tissue size and fat content in mice. Proc. Natl. Acad. Sci. U. S. A. 99:625-630.

22. Glorian, M., et al. 2001. A single element in the phosphoenolpyruvate carboxykinase gene mediates thiazolidinedione action specifically in adipocytes. Biochimie. 83:933-943.

23. Guan, H.P., et al. 2002. A futile metabolic cycle activated in adipocytes by antidiabetic agents. Nat. Med. 8:1122-1128.

24. Tan, G.D., et al. 2003. A "futile cycle" induced by thiazolidinediones in human adipose tissue? Nat. Med. 9:811-812; author reply 812.

25. Loviscach, M., et al. 2000. Distribution of peroxisome proliferator-activated receptors (PPARs) in human skeletal muscle and adipose tissue: relation to insulin action. Diabetologia. 43:304-311.

26. Auboeuf, D., et al. 1997. Tissue distribution and quantification of the expression of mRNAs of peroxisome proliferator-activated receptors and liver $\mathrm{X}$ receptor-alpha in humans: no alteration in adipose tissue of obese and NIDDM patients. Diabetes. 46:1319-1327.

27. Xu, H., et al. 2003. Chronic inflammation in fat plays a crucial role in the development of obesity-related insulin resistance. J. Clin. Invest. 112:1821-1830. doi:10.1172/JCI200318012.

28. Weisberg, S.P., et al. 2003. Obesity is associated with macrophage accumulation in adipose tissue. J. Clin. Invest. 112:1796-1808. doi:10.1172/ JCI200318012

29. Spiegelman, B.M., and Heinrich, R. 2004. Biological control through regulated transcriptional coactivators. Cell. 119:157-167.

30. Lehmann, J.M., et al. 1995. An antidiabetic thiazolidinedione is a high affinity ligand for peroxisome proliferator-activated receptor gamma (PPAR gamma). J. Biol. Chem. 270:12953-12956.

31. Inzucchi, S.E., et al. 1998. Efficacy and metabolic effects of metformin and troglitazone in type II diabetes mellitus. N. Engl. J. Med. 338:867-872.

32. Maggs, D.G., et al. 1998. Metabolic effects of troglitazone monotherapy in type 2 diabetes mellitus. A randomized, double-blind, placebo-controlled trial. Ann. Intern. Med. 128:176-185.

33. Frias, J.P., Yu, J.G., Kruszynska, Y.T., and Olefsky, J.M. 2000. Metabolic effects of troglitazone therapy in type 2 diabetic, obese, and lean normal subjects. Diabetes Care. 23:64-69.

34. Miyazaki, Y., et al. 2001. Improved glycemic control and enhanced insulin sensitivity in type 2 diabetic subjects treated with pioglitazone. Diabetes Care. 24:710-719.

35. Miyazaki, Y., et al. 2002. Effect of pioglitazone on abdominal fat distribution and insulin sensitivity in type 2 diabetic patients. J. Clin. Endocrinol. Metab. 87:2784-2791.

36. Nolan, J.J., Ludvik, B., Beerdsen, P., Joyce, M., and Olefsky, J. 1994. Improvement in glucose tolerance and insulin resistance in obese subjects treated with troglitazone. N. Engl. J. Med. 331:1188-1193.

37. Sironi, A.M., et al. 1997. Effects of troglitazone on insulin action and cardiovascular risk factors in patients with non-insulin-dependent diabetes. Clin. Pharmacol. Ther. 62:194-202.

38. Suter, S.L., Nolan, J.J., Wallace, P., Gumbiner, B., and Olefsky, J.M. 1992. Metabolic effects of new oral hypoglycemic agent CS-045 in NIDDM subjects. Diabetes Care. 15:193-203.

39. Mudaliar, S., Chang, A.R., and Henry, R.R. 2003. Thiazolidinediones, peripheral edema, and type 2 diabetes: incidence, pathophysiology, and clinical implications. Endocr. Pract. 9:406-416.
40. Guan, Y., et al. 2005. Thiazolidinediones expand body fluid volume through PPARgamma stimulation of ENaC-mediated renal salt absorption. Nat. Med. 11:861-866

41. Zhang, H., et al. 2005. Collecting duct-specific deletion of peroxisome proliferator-activated receptor gamma blocks thiazolidinedioneinduced fluid retention. Proc. Natl. Acad. Sci. U. S. A. 102:9406-9411.

42. Larsen, T.M., Toubro, S., and Astrup, A. 2003. PPARgamma agonists in the treatment of type II diabetes: is increased fatness commensurate with long-term efficacy? Int. J. Obes. Relat. Metab. Disord. 27:147-161.

43. van Harmelen, V., et al. 2002. Increased lipolysis and decreased leptin production by human omental as compared with subcutaneous preadipocytes. Diabetes. 51:2029-2036.

44. Sewter, C.P., Blows, F., Vidal-Puig, A., and O'Rahilly, S. 2002. Regional differences in the response of human pre-adipocytes to PPARgamma and RXRalpha agonists. Diabetes. 51:718-723.

45. Adams, M., et al. 1997. Activators of peroxisome proliferator-activated receptor $\gamma$ have depot-specific effects on human preadipocyte differentiation. J. Clin. Invest. 100:3149-3153.

46. Jensen, M.D. 1997. Lipolysis: contribution from regional fat. Annu. Rev. Nutr. 17:127-139.

47. Garg, A. 2004. Regional adiposity and insulin resistance. J. Clin. Endocrinol. Metab. 89:4206-4210.

48. Ye, J.M., et al. 2004. Direct demonstration of lipid sequestration as a mechanism by which rosiglitazone prevents fatty-acid-induced insulin resistance in the rat: comparison with metformin. Diabetologia. 47:1306-1313.

49. Raskin, P., et al. 2000. Rosiglitazone short-term monotherapy lowers fasting and post-prandial glucose in patients with type II diabetes. Diabetologia. 43:278-284.

50. Trujillo, M.E., and Scherer, P.E. 2005. Adiponectin: journey from an adipocyte secretory protein to biomarker of the metabolic syndrome. J. Intern. Med. 257:167-175

51. Weyer, C., et al. 2001. Hypoadiponectinemia in obesity and type 2 diabetes: close association with insulin resistance and hyperinsulinemia. J. Clin. Endocrinol. Metab. 86:1930-1935.

52. Yamamoto, Y., et al. 2002. Correlation of the adipocyte-derived protein adiponectin with insulin resistance index and serum high-density lipoprotein-cholesterol, independent of body mass index, in the Japanese population. Clin. Sci. (Lond.) 103:137-142.

53. Yu, J.G., et al. 2002. The effect of thiazolidinediones on plasma adiponectin levels in normal, obese, and type 2 diabetic subjects. Diabetes. 51:2968-2974.

54. Yang, W.S., et al. 2002. Plasma adiponectin levels in overweight and obese Asians. Obes. Res. 10:1104-1110.

55. Kern, P.A., Di Gregorio, G.B., Lu, T., Rassouli, N., and Ranganathan, G. 2003. Adiponectin expression from human adipose tissue: relation to obesity, insulin resistance, and tumor necrosis factoralpha expression. Diabetes. 52:1779-1785.

56. Maeda, N., et al. 2001. PPARgamma ligands increase expression and plasma concentrations of adiponectin, an adipose-derived protein. Diabetes. 50:2094-2099.

57. Yang, W.S., et al. 2002. Synthetic peroxisome proliferator-activated receptor-gamma agonist, rosiglitazone, increases plasma levels of adiponectin in type 2 diabetic patients. Diabetes Care. 25:376-380.

58. Combs, T.P., et al. 2004. A transgenic mouse with a deletion in the collagenous domain of adiponectin displays elevated circulating adiponectin and improved insulin sensitivity. Endocrinology. 145:367-383.

59. Yang, Q., et al. 2005. Serum retinol binding protein 4 
contributes to insulin resistance in obesity and type 2 diabetes. Proc. Natl. Acad. Sci. U. S. A. 36:356-362.

60. Yonemitsu, S., et al. 2001. Troglitazone induces GLUT4 translocation in L6 myotubes. Diabetes. 50:1093-1101.

61. Ciaraldi, T.P., Gilmore, A., Olefsky, J.M., Goldberg, M., and Heidenreich, K.A. 1990. In vitro studies on the action of CS-045, a new antidiabetic agent. Metabolism. 39:1056-1062.

62. Yang, Z., Strickland, D.K., and Bornstein, P. 2001. Extracellular matrix metalloproteinase 2 levels are regulated by the low density lipoprotein-related scavenger receptor and thrombospondin 2. J. Biol. Chem. 276:8403-8408.

63. Cha, B.S., et al. 2001. Peroxisome proliferator-activated receptor (PPAR) gamma and retinoid $X$ receptor (RXR) agonists have complementary effects on glucose and lipid metabolism in human skeletal muscle. Diabetologia. 44:444-452.

64. Kausch, C., et al. 2001. Effects of troglitazone on cellular differentiation, insulin signaling, and glucose metabolism in cultured human skeletal muscle cells. Biochem. Biophys. Res. Commun. 280:664-674.

65. Norris, A.W., et al. 2003. Muscle-specific PPAR $\gamma$ deficient mice develop increased adiposity and insulin resistance but respond to thiazolidinediones. J. Clin. Invest. 112:608-618. doi:10.1172/ JCI200317305.

66. Hevener, A.L., et al. 2003. Muscle-specific Pparg deletion causes insulin resistance. Nat. Med. 9:1491-1497.

67. Burant, C.F., et al. 1997. Troglitazone action is independent of adipose tissue. J. Clin. Invest. 100:2900-2908.

68. Edvardsson, U., et al. 1999. Rosiglitazone (BRL49653), a PPARgamma-selective agonist, causes peroxisome proliferator-like liver effects in obese mice. J. Lipid Res. 40:1177-1184.

69. Memon, R.A., et al. 2000. Up-regulation of peroxisome proliferator-activated receptors (PPAR-alpha) and PPAR-gamma messenger ribonucleic acid expression in the liver in murine obesity: troglitazone induces expression of PPAR-gamma-responsive adipose tissue-specific genes in the liver of obese diabetic mice. Endocrinology. 141:4021-4031.

70. Bedoucha, M., Atzpodien, E., and Boelsterli, U.A. 2001. Diabetic KKAy mice exhibit increased hepatic PPARgamma1 gene expression and develop hepatic steatosis upon chronic treatment with antidiabetic thiazolidinediones. J. Hepatol. 35:17-23.

71. Rahimian, R., et al. 2001. Hepatic over-expression of peroxisome proliferator activated receptor gamma2 in the ob/ob mouse model of non-insulin dependent diabetes mellitus. Mol. Cell. Biochem. 224:29-37.

72. Yu, S., et al. 2003. Adipocyte-specific gene expression and adipogenic steatosis in the mouse liver due to peroxisome proliferator-activated receptor gamma1 (PPARgamma1) overexpression. J. Biol. Chem. 278:498-505.

73. Neuschwander-Tetri, B.A., et al. 2003. Interim results of a pilot study demonstrating the early effects of the PPAR-gamma ligand rosiglitazone on insulin sensitivity, aminotransferases, hepatic steatosis and body weight in patients with nonalcoholic steatohepatitis. J. Hepatol. 38:434-440.

74. Sanyal, A.J., et al. 2004. A pilot study of vitamin $\mathrm{E}$ versus vitamin $\mathrm{E}$ and pioglitazone for the treatment of nonalcoholic steatohepatitis. Clin. Gastroenterol. Hepatol. 2:1107-1115.

75. Promrat, K., et al. 2004. A pilot study of pioglitazone treatment for nonalcoholic steatohepatitis. Hepatology. 39:188-196.

76. Barroso, I., et al. 1999. Dominant negative mutations in human PPARgamma associated with severe insulin resistance, diabetes mellitus and hypertension. Proc. Natl. Acad. Sci. U. S. A. 402:880-883.

77. Agarwal, A.K., and Garg, A. 2002. A novel hetero- zygous mutation in peroxisome proliferator-activated receptor-gamma gene in a patient with familial partial lipodystrophy. J. Clin. Endocrinol. Metab. 87:408-411.

78. Hegele, R.A., Cao, H., Frankowski, C., Mathews, S.T., and Leff, T. 2002. PPARG F388L, a transactivation-deficient mutant, in familial partial lipodystrophy. Diabetes. 51:3586-3590.

79. Agostini, M., et al. 2004. Tyrosine agonists reverse the molecular defects associated with dominantnegative mutations in human peroxisome proliferator-activated receptor gamma. Endocrinology. 145:1527-1538.

80. Mori, Y., et al. 1999. Effect of troglitazone on body fat distribution in type 2 diabetic patients. Diabetes Care. 22:908-912

81. Kelly, I.E., Han, T.S., Walsh, K., and Lean, M.E. 1999. Effects of a thiazolidinedione compound on body fat and fat distribution of patients with type 2 diabetes. Diabetes Care. 22:288-293.

82. Misra, A., Peethambaram, A., and Garg, A. 2004. Clinical features and metabolic and autoimmune derangements in acquired partial lipodystrophy: report of 35 cases and review of the literature. Medicine (Baltimore). 83:18-34.

83. Savage, D.B., et al. 2003. Human metabolic syndrome resulting from dominant-negative mutations in the nuclear receptor peroxisome proliferator-activated receptor-gamma. Diabetes. 52:910-917.

84. Savage, D.B., Murgatroyd, P.R., Chatterjee, V.K., and O'Rahilly, S. 2005. Energy expenditure and adaptive responses to an acute hypercaloric fat load in humans with lipodystrophy. J. Clin. Endocrinol. Metab. 90:1446-1452.

85. Nakamura, Y., et al. 1998. Inhibitory action of insulin-sensitizing agents on calcium channels in smooth muscle cells from resistance arteries of guinea-pig. Br. J. Pharmacol. 123:675-682.

86. Satoh, H., et al. 1999. Thiazolidinediones suppress endothelin-1 secretion from bovine vascular endothelial cells: a new possible role of PPARga$\mathrm{mma}$ on vascular endothelial function. Biochem. Biophys. Res. Commun. 254:757-763.

87. Tsai, Y.S., et al. 2004. Hypertension and abnormal fat distribution but not insulin resistance in mice with P465L PPAR $\gamma$. J. Clin. Invest. 114:240-249. doi:10.1172/JCI200318012.

88. Freedman, B.D., Lee, E.J., Park, Y., and Jameson, J.L. 2005. A dominant negative peroxisome proliferator-activated receptor-gamma knock-in mouse exhibits features of the metabolic syndrome. J. Biol. Chem. 280:17118-17125.

89. Semple, R.K., et al. 2005. A dominant negative human peroxisome proliferator-activated receptor (PPAR)alpha is a constitutive transcriptional corepressor and inhibits signaling through all PPAR isoforms. Endocrinology. 146:1871-1882.

90. Savage, D.B., et al. 2002. Digenic inheritance of severe insulin resistance in a human pedigree. Nat. Genet. 31:379-384.

91. Kubota, N., et al. 1999. PPAR gamma mediates high-fat diet-induced adipocyte hypertrophy and insulin resistance. Mol. Cell. 4:597-609.

92. Miles, P.D., Barak, Y., He, W., Evans, R.M., and Olefsky, J.M. 2000. Improved insulin-sensitivity in mice heterozygous for PPAR- $\gamma$ deficiency. J. Clin. Invest. 105:287-292.

93. Altshuler, D., et al. 2000. The common PPARgamma Pro12Ala polymorphism is associated with decreased risk of type 2 diabetes. Nat. Genet. 26:76-80.

94. Stumvoll, M., and Haring, H. 2002. The peroxisome proliferator-activated receptor-gamma2 Pro12Ala polymorphism. Diabetes. 51:2341-2347.

95. Deeb, S.S., et al. 1998. A Pro12Ala substitution in PPARgamma2 associated with decreased receptor activity, lower body mass index and improved insu- lin sensitivity. Nat. Genet. 20:284-287.

96. Masugi, J., Tamori, Y., Mori, H., Koike, T., and Kasuga, M. 2000. Inhibitory effect of a proline-toalanine substitution at codon 12 of peroxisome proliferator-activated receptor-gamma 2 on thiazolidinedione-induced adipogenesis. Biochem. Biophys. Res. Commun. 268:178-182.

97. Poulsen, P., et al. 2003. Impact of two common polymorphisms in the PPARgamma gene on glucose tolerance and plasma insulin profiles in monozygotic and dizygotic twins: thrifty genotype, thrifty phenotype, or both? Diabetes. 52:194-198.

98. Andrulionyte, L., Zacharova, J., Chiasson, J.L., and Laakso, M. 2004. Common polymorphisms of the PPAR-gamma2 (Pro12Ala) and PGC-1alpha (Gly482Ser) genes are associated with the conversion from impaired glucose tolerance to type 2 diabetes in the STOP-NIDDM trial. Diabetologia. 47:2176-2184.

99. Memisoglu, A., et al. 2003. Prospective study of the association between the proline to alanine codon 12 polymorphism in the PPARgamma gene and type 2 diabetes. Diabetes Care. 26:2915-2917.

100.Ek, J., et al. 2001. Studies of the Pro12Ala polymorphism of the peroxisome proliferator-activated receptor-gamma2 (PPAR-gamma2) gene in relation to insulin sensitivity among glucose tolerant caucasians. Diabetologia. 44:1170-1176.

101.Zouari Bouassida, K., et al. 2005. The peroxisome proliferator activated receptorgamma2 (PPARgamma2) Pro12Ala variant: lack of association with type 2 diabetes in obese and non obese Tunisian patients. Diabetes Metab. 31:119-123.

102.Malecki, M.T., et al. 2003. The Pro12Ala polymorphism of PPARgamma2 gene and susceptibility to type 2 diabetes mellitus in a Polish population. Diabetes Res. Clin. Pract. 62:105-111.

103.Poirier, O., Nicaud, V., Cambien, F., and Tiret, L. 2000. The Pro12Ala polymorphism in the peroxisome proliferator-activated receptor gamma 2 gene is not associated with postprandial responses to glucose or fat tolerance tests in young healthy subjects: the European Atherosclerosis Research Study II. J. Mol. Med. 78:346-351.

104.Oh, E.Y., et al. 2000. Significance of Pro12Ala mutation in peroxisome proliferator-activated receptorgamma 2 in Korean diabetic and obese subjects. J. Clin. Endocrinol. Metab. 85:1801-1804.

105. Cole, S.A., et al. 2000. The Pro12Ala variant of peroxisome proliferator-activated receptor-gamma2 (PPAR-gamma2) is associated with measures of obesity in Mexican Americans. Int. J. Obes. Relat. Metab. Disord. 24:522-524.

106.Beamer, B.A., et al. 1998. Association of the Pro$12 \mathrm{Ala}$ variant in the peroxisome proliferator-activated receptor-gamma2 gene with obesity in two Caucasian populations. Diabetes. 47:1806-1808.

107. Valve, R., et al. 1999. Two polymorphisms in the peroxisome proliferator-activated receptor-gamma gene are associated with severe overweight among obese women. J. Clin. Endocrinol. Metab. 84:3708-3712.

108. Meirhaeghe, A., Cottel, D., Amouyel, P., and Dallongeville, J. 2005. Association between peroxisome proliferator-activated receptor gamma haplotypes and the metabolic syndrome in French men and women. Diabetes. 54:3043-3048.

109. Meirhaeghe, A., et al. 2005. Study of a new PPARgamma2 promoter polymorphism and haplotype analysis in a French population. Mol. Genet. Metab. 85:140-148.

110.Doney, A., et al. 2002. Haplotype analysis of the PPARgamma Pro12Ala and C1431T variants reveals opposing associations with body weight. BMC Genet. 3:21.

111. Memisoglu, A., et al. 2003. Interaction between a peroxisome proliferator-activated receptor gamma gene polymorphism and dietary fat intake in relation to body mass. Hum. Mol. Genet. 12:2923-2929. 
112.Robitaille, J., Despres, J.P., Perusse, L., and Vohl, M.C. 2003. The PPAR-gamma P12A polymorphism modulates the relationship between dietary fat intake and components of the metabolic syndrome: results from the Quebec Family Study. Clin. Genet. 63:109-116.

113.Luan, J., et al. 2001. Evidence for gene-nutrient interaction at the PPARgamma locus. Diabetes. 50:686-689.

114.Nicklas, B.J., et al. 2001. Genetic variation in the peroxisome proliferator-activated receptor-gamma2 gene (Pro12Ala) affects metabolic responses to weight loss and subsequent weight regain. Diabetes. 50:2172-2176.

115.Lindi, V., et al. 2003. Impact of the Pro12Ala polymorphism of the PPAR-gamma2 gene on serum triacylglycerol response to n-3 FA supplementation. Mol. Genet. Metab. 79:52-60.

116. Adamo, K.B., et al. 2005. Influence of Pro12Ala peroxisome proliferator-activated receptor gamma2 polymorphism on glucose response to exercise train- ing in type 2 diabetes. Diabetologia. 48:1503-1509. 117. Ostergard, T., et al. 2005. Influence of the PPARgamma2 Pro12Ala and ACE I/D polymorphisms on insulin sensitivity and training effects in healthy offspring of type 2 diabetic subjects. Horm. Metab. Res. 37:99-105.

118. Weiss, E.P., et al. 2005. Endurance training-induced changes in the insulin response to oral glucose are associated with the peroxisome proliferator-activated receptor-gamma2 Pro12Ala genotype in men but not in women. Metabolism. 54:97-102.

119.Kahara, T., et al. 2003. PPARgamma gene polymorphism is associated with exercise-mediated changes of insulin resistance in healthy men. Metabolism. 52:209-212.

120.Franks, P.W., et al. 2004. Does peroxisome proliferator-activated receptor gamma genotype (Pro12ala) modify the association of physical activity and dietary fat with fasting insulin level? Metabolism. 53:11-16.

121.Tschritter, O., et al. 2003. Increased insulin clear- ance in peroxisome proliferator-activated receptor gamma2 Pro12Ala. Metabolism. 52:778-783.

122.Stumvoll, M., et al. 2001. Pro12Ala polymorphism in the peroxisome proliferator-activated receptorgamma2 gene is associated with increased antilipolytic insulin sensitivity. Diabetes. 50:876-881.

123.Schneider, J., Kreuzer, J., Hamann, A., Nawroth, P.P., and Dugi, K.A. 2002. The proline 12 alanine substitution in the peroxisome proliferator-activated receptor-gamma2 gene is associated with lower lipoprotein lipase activity in vivo. Diabetes. 51:867-870.

124. Mousavinasab, F., et al. 2005. Common polymorphisms in the PPARgamma 2 and IRS- 1 genes and their interaction influence serum adiponectin concentration in young Finnish men. Mol. Genet. Metab. 84:344-348.

125. Takata, N., et al. 2004. Pro12Ala substitution in peroxisome proliferator-activated receptor gamma 2 is associated with low adiponectin concentrations in young Japanese men. Metabolism. 53:1548-1551. 